\title{
Seelsorger im Abseits der Massenkommunikation ${ }^{1}$
}

\author{
von Jacob Gerard Stappers
}

Seit ein paar Jahren sind nicht mehr nur die gewöhnlichen Sterblichen Gegenstand ernsthafter sozial-wissenschaftlicher Forschung. Auch die Diener des Wortes, ihre Aktivitäten sowie Struktur und Funktion ihrer Organisationen werden empirisch untersucht. Von daher gesehen ist es nicht verwunderlich, daß einzelne katholische Organisationen, die im Bereich der Massenkommunikation arbeiten oder damit zu tun haben, eine Untersuchung über das Verhalten des katholischen Pfarrers, im weiteren Sinne: des praktischen Seelsorgers, zu verschiedenen Erscheinungsformen der Massenkommunikation unternommen haben.

Eine der Hauptfragen, die zu Kommunikation und Massenkommunikation gestellt werden, zielt $a b$ auf Gelingen und Versagen oder, wenn man so will, auf den Erfolg von Kommunikation und Massenkommunikation; für die Massenkommunikation suchte und "fand" man eine Antwort darauf unter dem Aspekt der Beeinflussungsmöglichkeit der Massenmedien, populärer ausgedrückt der "Macht der Medien“. Bis vor kurzem war es selbstverständlich, die "Macht“ so zu verteilen, daß die SenderMedium-Seite gegenüber der Empfänger-Seite eindeutig die Oberhand hatte. In dieser traditionellen Sicht auf den Einfluß der Massenkommunikation wurde der Rezipient allenfalls als Durchgangsstation der Wirkung oder als ein Knotenpunkt intervenierender, die Wirkung eingrenzender Variablen verstanden.

Diese und verwandte Sichtweisen auf die Wirkmöglichkeit der Massenkommunikation, zusammengefaßt unter dem Etikett "hypodermic-needle-theory" 2 (InjektionsnadelTheorie) oder auch „transmissionbelt-theory "3 (Transmissionsriemen-Theorie), werden durch die sozial-wissenschaftliche Forschung nicht gestützt. ${ }^{4}$ Es waren Untersuchungen aus dem Beginn der vierziger Jahre, die einen ersten Ansatz dazu gaben, über die Selbstverständlichkeit des Einflusses der Massenkommunikation Überlegungen anzustellen. Derartige sozialwissenschaftliche Untersuchungen erschütterten die Vorstellung von den allmächtigen Medien, „entdeckten“ hingegen das Faktum der Beeinflussung durch „andere ${ }^{*}$ (personal influence).

Auf dieser Grundlage formulierten Katz und Lazarsfeld ${ }^{3}$ ihre berühmte "two-stepflow-of-communication "-Hypothese (Zwei-Stufen-Weg der Kommunikation): der Einfluß der Massenmedien ist nicht direkt, sondern verläuft in zwei Schritten, erst zu den „opinion leaders“ (Meinungsführern), und über sie werden dann die anderen Menschen, die „Nach-Folger“, beeinflußt.

Katz und Lazarsfeld bewirkten mit dieser Hypothese einen definitiven Bruch mit der Theorie von den allmächtigen Medien. Tatsächlich stellt die Hypothese nur fest, daß die innere Struktur des Beeinflussungsprozesses aus mehr als einem Schritt besteht und komplizierter strukturiert ist, als man zunächst dachte. Wird die Hypothese im strengen Sinn verstanden, dann wird der vom Sender-Medien-Komplex als solchem ausgehende Einfluß nicht bezweifelt. ${ }^{6}$ Soviel zur begrifflichen Vorklärung.

Dr. Jacob Gerard Stappers ist Lektor für Publizistik- und Kommunikationswissenschaft am Instituut voor Massacommunicatie der Katholischen Universität Nimwegen/Niederlande. 
Die Beziehung Seelsorger-Massenkommunikation kann von mehreren Seiten aus angegangen werden. Im Anschluß an das Gesagte kann man sich fragen, wie und auf welche Weise der Pfarrer als "gate-keeper" (Schleusenwärter) in dem durch massenkommunikative Kommunikator-Instanzen veranlaßten Strom der Informationen (flow of information) fungiert.

Unsere Darstellung wird sich jedoch auf eine andere Frage beschränken. Wenn man die Arbeit der Seelsorger als "agogisch" charakterisiert, d. h. als das Begleiten von Menschen namentlich da, wo es um die religiöse Dimension ihres Lebens geht, wenn man weiterhin feststellt, daß sich dieses Begleiten vornehmlich seitens der Seelsorger in einer großen Verschiedenheit von Kommunikations-Aktivitäten realisiert, publizistisch bis persönlich der Art nach, direkt bis indirekt auf die pastorale Arbeit bezogen, kann man sich fragen, inwiefern etwa ein Pfarrer für die Menschen, für die er arbeitet, ein ernsthafter Gesprächspartner auf dem Sachgebiet des täglich wiederkehrenden massenkommunikativen Nachrichtenangebots ist.

Diese Frage ist gewichtiger, als sie auf den ersten Blidk zu sein sheint. Vorsichtig geschätzt verwendet der "Durchschnitts-Niederländer“ pro Durchschnittstag durchschnittlich mindestens fünf Stunden auf dieses täglich wiederkehrende massenkommunikative Angebot: Zeitunglesen, Fernsehen und Rundfunkhören. (Der „Durchschnitts-Deutsche" [B.R.D.] begnügt sich mit drei Stunden und 34 Minuten.) ${ }^{7}$

Zeitunglesen, Fernsehen und Rundfunkhören gehören zu den Selbstverständlichkeiten des Lebens aller Tage und nehmen nach dem Schlafen und Arbeiten die meiste Zeit in Anspruch. Das bedeutet allerdings noch nicht, daß die Massenmedien ohne weiteres sehr „einflußreich“ sind.

Der Empfangssituation kann ein bedeutendes $M a ß$ an Freiwilligkeit auf Seiten des Rezipienten nicht abgesprochen werden. Die Rezeptionssituation im Massenkommunikationsprozeß ist derart, daß der Rezipient Spielraum behält, sich einer Nachricht in Hinsicht auf Interpretation, Verarbeitung und auch auf das Weiter-wirken-Lassen einer Nachricht in seinem eigenen personalen System auszusetzen oder nicht. Stephenson $^{8}$ charakterisiert diese Möglichkeiten des Rezipienten als Äußerungen von „convergent selectivity": ", in which the object is to let each person choose something different for himself " ${ }^{\text {. }}$ "Convergent selectivity " steht mehr oder minder dem Ansatz von "social control" gegenüber. Soziale Kontrolle manifestiert sich in Uberzeugungen, Werten und Normen, die Menschen in gewissem Sinn zwingen, sich in bestimmter Weise zu verhalten. Rezipieren ist nicht ein Geprägt-Werden durch eines anderen Nachricht, sondern aktiv-reaktive Sinngebung. Einerseits erklärt dies den "Mangel“ an Erfolg, andererseits das Ubermaß an Emotionen und Ergriffenheit bei denen, die sich in ihrem personalen System angesprochen fühlen. Es ist der Umkreis subjektiv erlebten Spielraums, der beide Reaktionen ermöglicht, ohne daß sie einander widerstreiten. Es ist hiermit jedoch die Möglichkeit massenkommunikativen Angebots angezeigt, das das Individuum weit außerhalb der geeichten Pfade seines Alltagslebens und seiner gebräuchlichen Sinngebung führen kann, unabhängig davon, ob das Angebot "Een van de Acht", „Bonanza“", "Peyton Place" oder "Kenmerk" betrifft."

Umgang mit den Massenmedien spielt sich nach diesem Verständnis nicht am Rand des Alltagslebens des Individuums ab, sondern ist zumindest quantitativ ein wichtiger Bestandteil dieses Lebens und verschafft darüber hinaus die Möglichkeit, die eigene Individualität und die damit zusammenhängende Problematik im persönlichen und subjektiven Reagieren auf das Informationsangebot zu erleben. 
Wenn wir nun auf die Frage zurückgreifen, ob der Seelsorger ein ernsthafter Gesprächspartner in Sachen des massenkommunikativen Angebots ist, kann man sich mehr im besonderen fragen, ob er vom Rezeptionsverhalten von der übergroßen Mehrheit der Menschen, für die er tätig ist, abweicht, ob er auf dem Gebiet des Umgangs mit den Massenmedien abseits steht und isoliert ist vom Durchschnittsgläubigen und ob vielleicht sein Rezeptionsverhalten elitäre Züge trägt?

Zur Beantwortung dieser Fragen zunächst eine kurze Öbersicht über die sozialempirische Untersuchung PASTOMAC ${ }^{10}$ und einige ihrer Ergebnisse.

\section{Allgemeines}

Stichprobe: Die Untersuchungsgrundgesamtheit sind die niederländischen katholischen Seelsorger, die in der Basisseelsorge arbeiten, die früher als "parochiale Seelsorge" bezeichnet wurde. Hierunter fallen: Dechanten, Hilfsdechanten, Dekanatspfarrer, Pfarrer, "Deservitores“, Vikare oder "Economici“, Rektoren von Hilfskirchen, Kapläne, Assistenten, Dekanats-Assistenten und Pfarrer, die als Leiter oder Mitarbeiter in einem Dekanats-, interparochialen oder parochialen Team arbeiten. Aus der gesamten genannten Population, nach den Namenslisten der Bistümer per 1. Januar 1971 gut 3395 Seelsorger, wurde eine zufällige Stichprobe von 350 und eine Reserve-Stichprobe von 110 ausgewählt. Die mündlichen Interviews anhand eines großenteils vorgefertigten Fragebogens wurden in der Zeit vom 6. bis 17. Januar 1971 durchgeführt.

Um an die gewünschte Stichprobengröße von 350 möglichst nahe heranzukommen, wurden insgesamt 420 Seelsorger angesprochen. So ergaben sich 312 vollständige Fragegespräche. Die Nichtbeantwortung beläuft sich auf 25,7 Prozent, der Prozentsatz der echten Verweigerung auf 13,8 Prozent. In Anbetracht der kurzen Zeit, in der die Interviews stattfinden mußten, kann eine Antwortquote von 74,3 Prozent als befriedigend angesehen werden. Aufgrund eines Vergleichs bekannter Personaldaten der Grundgesamtheit mit der der befragten Seelsorgergruppe (Lebenszeit, Ordenspriester, Weltpriester, Funktion, Bistum) kann man behaupten, daß die tatsächlich befragte Pfarrergruppe für die Gesamtheit hinreichend repräsentativ ist. Die Stichprobeneinheit ist der Seelsorger in der Basisseelsorge. Die Ergebnisse der Untersuchung können also nur auf diese Grundgesamtheit hin verallgemeinert werden und nicht auf andere Sektoren oder auf die Gesamtpopulation der niederländischen Priester.

Strukturelle Gegebenheiten: Die in der Basisseelsorge arbeitenden Priester sind eine ziemlich überalterte Berufsgruppe: nur 17,5 Prozent sind jünger als 40 Jahre, 24,2 Prozent befinden sich im Lebensalter zwischen 40 und 49 Jahren, 55 Prozent sind 50 Jahre oder älter. Der Priestermangel in der Basisseelsorge spiegelt sich auch im Einsatz von Ordenspriestern wider, vor allem in der Diasporadiözese Groningen, wo die Hälfte der besetzten Stellen von Ordenspriestern eingenommen wird. In den

* „Een van de Acht“ ist ein populäres Quizprogramm in den Niederlanden, das während der Wintersaison einmal monatlich am Samstagabend ausgestrahlt wird, zu vergleichen mit "Einer wird gewinnen". "Bonanza" ist die auch in Deutschland bekannte amerikanische Wildwestserie; "Peyton Place“ ist ebenfalls eine amerikanische Fernsehserie. Diese Programme werden als Höhepunkte kleinbürgerlicher Ergötzlichkeit interpretiert. "Kenmerk" ist ein kritisches Informationsprogramm des niederländischen Fernsehens über kirchliche Angelegenheiten. 
anderen Bistümern ist ungefähr ein Drittel der Stellen jeweils von Angehörigen eines religiösen Ordens oder einer Kongregation besetzt. Es besteht eine starke Korrelation zwischen Lebensalter und Funktion: 55 Prozent der Beantworter gehören zu den höheren Amtsträgern (Dechant, Dechant-Pfarrer, Deservitor, Teamleiter, Rektor); von den jüngeren Pfarrern (unter 45 Jahren) haben nur 16 Prozent eine derartige Funktion, gegenüber 79 Prozent der mittleren Altersgruppe (45 bis 54 Jahre) und 73 Prozent der höheren Altersgruppe (55 Jahre und älter).

Im Rahmen der oben gestellten Fragen ist es relevant, global zu wissen, in welchem Milieu die Menschen leben, für die die Seelsorger tätig sind. Zwei Drittel der Befragten sagten aus, daß die Menschen, für die sie arbeiten, zur unteren und zur Mittelschicht gehören, bei 31,4 Prozent überwiegt keine einzelne Schicht, und 1,6 Prozent erklären, überwiegend mit Menschen der Oberschicht zu tun zu haben. Hiermit besteht eine erste Diskrepanz zwischen den Priestern und den Menschen, für die sie arbeiten; der Priester selbst ist hinsichtlich seines Erziehungsmilieus zur Oberschicht ${ }^{11}$ zu rechnen. Faktoren, die das Rezeptionsverhalten des Pfarrers mitbestimmen können, sind: seine Wohnsituation und die Zeit, dic er seiner Arbeit widmet. Der Priester, der mit Kollegen zusammenwohnt, wird möglicherweise in seiner freien Wahl hinsichtlich Rundfunk- und/oder Fernsehangebot eingeengt, während andererseits die Möglichkeit, Tages- und Wochenzeitungen, Zeitschriften und Bücher auszutauschen, vergrößert wird. 33 Prozent der Seelsorger wohnen allein, 26 Prozent mit einem Priester und 41 Prozent mit mehr als einem Priester zusammen. Das Allein-Wohnen kommt in Gemeinden auf dem flachen Land und bei Seelsorgern mit einer hohen Funktion am meisten vor.

Priester sind stark in Anspruch genommen, und vor allem durch ihre (mögliche) Abendarbeit weichen sie in ihrem Freizeitverhalten vom Durchschnitts-Niederländer ab. Unsere Untersuchung entschied sich dafür, den Seelsorger selbst die wirkliche Dauer seiner Arbeitswoche schätzen zu lassen. Diese Entscheidung wurde darum ge-

\begin{tabular}{l|c|c}
\hline Durchschnittszahl der Arbeitsstunden pro Woche & $\begin{array}{c}\text { Prozent } \\
\text { der Befragten }\end{array}$ & $\begin{array}{c}\text { absolut } \\
\text { der Befragten }\end{array}$ \\
\hline 10 Stunden oder weniger & 2,6 & 8 \\
11 bis 20 Stunden & 2,9 & 9 \\
21 bis 30 Stunden & 2,2 & 7 \\
31 bis 40 Stunden & 1,6 & 5 \\
41 bis 50 Stunden & 14,4 & 45 \\
51 bis 60 Stunden & 23,7 & 74 \\
61 bis 70 Stunden & 23,4 & 73 \\
71 Stunden und mehr & 15,1 & 47 \\
weiß nicht & 13,5 & 42 \\
keine Antwort & 0,6 & 2 \\
\hline Gesamt & 100 & 312 \\
\hline
\end{tabular}


troffen, weil unterstellt wurde, daß der subjektive Eindruck, den der Pfarrer vom Umfang seiner Arbeitswoche hat und vom Maß, in dem er unter Druck steht, für seinen Umgang mit den Massenmedien bestimmender ist als die objektiv meßbare Zeit, die ihm hierfür zur Verfügung steht: Man übt nicht deshalb Fernseh-Abstinenz, weil man objektiv nicht die Zeit dazu hat, sondern weil man sich aus subjektiv erlebtem Druck nicht die Zeit dafür nimmt.

29 Seelsorger (9 Prozent) geben eine Arbeitswoche an, die unter 41 Stunden liegt Das kann der Tatsache zugeschrieben werden, daß ein Teil der von den Bistümerı benannten Priester, die in der Basisseelsorge tätig sind, nur eine Teilaufgabe erfüllen, eventuell noch eine gewisse Ausbildung mitmachen oder hauptamtlich woanders tätig sind (z. B. im Unterricht).

Es gibt nur geringe Unterschiede zwischen den Lebensalterskategorien. Wohl fällt auf, daß von den 44 Seelsorgern, die auf die Frage nach der Dauer ihrer Arbeitswoche keine Antwort gaben, 36 (= 81 Prozent) keinen besonderen pastoralen Auftrag haben, während dieser Prozentsatz für die gesamte Stichprobe 72 Prozent beträgt. Das könnte ein Anzeichen dafür sein, daß das Vorhandensein eines besonderen pastoralen Auftrags leichter ein "timing " der Arbeitswoche mit sich bringt.

\section{Medien}

Fernsehen: 6 Priester sitzen nie vor dem Fernsehgerät, und bei einem von diesen befindet sich gar kein Fernsehapparat im Haus. Außerdem gibt es noch 5 Seelsorger, bei denen kein Fernsehapparat in ihrer Wohnung vorhanden ist.

Der Seelsorger widmet dem Fernsehen im Vergleich zum Durchschnitts-Niederländer besonders wenig Zeit. 76 Prozent sehen durchschnittlich eine Stunde oder weniger pro Tag, während der Durchschnitt für den Durchschnitts-Niederländer bei 2 bis 2,5 Stunden liegt. ${ }^{12}$ Der Seelsorger sieht auch weniger als der Durchschnitts-Niederländer mit mittlerer und/oder höherer Schulbildung. ${ }^{13} 44$ Prozent der Befragten sehen abends gewöhnlich nur vor $9 \mathrm{Uhr}$, während 16 Prozent gewöhnlich nur nach 9 Uhr sehen. NOS-Untersuchungen ${ }^{14}$ verzeichnen die Tendenz, daß sich unter den "späten “15 Sehern relativ mehr Menschen mit höherer Schulbildung finden als unter den "frühen " Sehern. Der Seelsorge-Priester fällt aus diesem Rahmen heraus.

Es besteht ein deutlicher Zusammenhang zwischen Lebensalter und Zeit, die dem Fernsehen gewidmet wird.

64 Prozent der jüngeren Seelsorger wendet eine halbe Stunde oder weniger pro Tag für Fernsehen auf, gegenüber 42 Prozent bei den 45- bis 54jährigen und 35 Prozent bei den Befragten im Alter von 55 Jahren und mehr. Der wichtigste Grund, den sie selbst für ihren (im Vergleich mit anderen) minimalen Umgang mit dem Fernsehen angeben, ist ihr Mangel an Zeit: Von den 154, die sagten, "selten" fernzusehen, nannten 113 als Grund „keine Zeit", 24 „nicht zu Hause“ und 17 einen anderen Grund wie "kein Fernsehgerät im Haus", "kein Interesse" oder "schlechte Programme". Hiermit stimmt überein der Zusammenhang zwischen der dem Fernsehen gewidmeten Zeit und der Durchschnittszahl der Arbeitsstunden pro Woche: je größer der Umfang der Arbeitswoche, umso weniger Zeit wird auf das Fernsehen verwendet (Korrelation: $r=-.42$ ). 
Dem Fernsehen gewidmete Zeit und Lebensalter

\begin{tabular}{|c|c|c|c|c|c|}
\hline \multirow{2}{*}{$\begin{array}{l}\text { Dem Fernsehen } \\
\text { gewidmete Zeit }\end{array}$} & \multicolumn{3}{|c|}{ Lebensalter } & \multirow{2}{*}{$\begin{array}{c}\text { Gesamt; } \\
\text { Prozent- } \\
\text { wert }\end{array}$} & \multirow{2}{*}{$\begin{array}{l}\text { Gesamt; } \\
\text { absolut }\end{array}$} \\
\hline & $\begin{array}{l}\text { bis } \\
44 \text { Jahre }\end{array}$ & $\begin{array}{l}45 \text { bis } \\
54 \text { Jahre }\end{array}$ & $\begin{array}{l}55 \mathrm{~J} \text {. } \\
\text { und älter }\end{array}$ & & \\
\hline 15 Min. od. weniger & 26,6 & 16,2 & 16,4 & 19,9 & 62 \\
\hline 16 bis 30 Min. & 37,6 & 26,3 & 19,2 & 27,9 & 87 \\
\hline 31 Min. bis 1 Std. & 20,2 & 38,4 & 26,9 & 28,2 & 88 \\
\hline 1 Std. bis $11 / 2$ Std. & 7,3 & 10,1 & 11,5 & 9,6 & 30 \\
\hline mehr als $11 / 2$ Std. & 6,4 & 6,1 & 16,3 & 9,6 & 30 \\
\hline keine Antwort & 1,8 & 3,0 & 9,6 & 4,8 & 15 \\
\hline Gesamt & $\begin{array}{c}99,9 \\
(109)\end{array}$ & $\begin{array}{l}100,1 \\
(99)\end{array}$ & $\begin{array}{c}99,9 \\
(104)\end{array}$ & 100 & 312 \\
\hline
\end{tabular}

Auf die Frage nach Programm-Arten, die er regelmäßig verfolgt, nennt der Seelsorger nicht jene, die das durchschnittliche niederländische Fernsehpublikum am meisten ansieht, etwa Filmserien und Unterhaltungsprogramme ${ }^{16}$, viel häufiger hingegen "religiöse Sendungen ", die gerade zu den am wenigsten verfolgten Programmen gehören. ${ }^{17}$ Die einzige Ausnahme bildet der Fußball; er ist bei den Priestern genau so beliebt wie bei der niederländischen Bevölkerung allgemein. Aus sämtlichen Antworten der Pfarrer ergibt sich vor allem ihre Aufmerksamkeit für Nachrichten, Aktualitäten und "schwere" Information sowie gesellschaftliche Dokumentationen. Das steht in starkem Kontrast zum Verhaltensmuster des durchschnittlichen Niederländers, wie die Nationaluntersuchung zeigt. ${ }^{18}$

Im allgemeinen verwenden Menschen mit hohem Bildungsniveau weniger Zeit auf das Fernsehen, aber eine Untersuchung, die vor einigen Jahren unter praktischen Ärzten ${ }^{19}$ durchgeführt wurde, zeigte auch, daß ihr Verhalten gegenüber regelmäßig verfolgten Programmen von dem des durchschnittlichen Niederländers kaum abwich. Der Hausarzt sah wenig, aber er verteilte sein Augenmerk gegenüber den verschiedenen Programmsparten auf dieselbe Weise wie der durchschnittliche Niederländer.

Rundfunk: Der durchschnittliche Niederländer im Alter von 12 Jahren und mehr hört tagsüber (zwischen 7 Uhr morgens und 7 Uhr abends) 3 Stunden Rundfunk. ${ }^{20}$ Von den befragten Seelsorgern hören 29 nie Rundfunk, 172 (= 55 Prozent) durchschnittlich eine halbe Stunde oder weniger, 36 (= 12 Prozent) $z$ wischen einer halben und einer ganzen Stunde, $54 \mathrm{mehr}$ als eine Stunde täglich. Auch themenmäßig weicht die dem Rundfunk gewidmete Zeit beim Priester stark vom durchschnittlichen Niederländer ab. Auf die Frage nach den Gründen, warum sie "selten" Rundfunk hören, erklären 96 Seelsorger, daß sie "keine Zeit" haben; 22, daß sie kein Interesse haben und 14, daß sie nicht zu Hause sind. Es scheint jedoch kein direkter Zusammenhang zu bestehen zwischen dem Umfang der Arbeitswoche und der Zeit, die man im Durchschnitt täglich auf das Rundfunkhören verwendet. Im Gegensatz zu dem, was das Sehverhalten angeht, wo Unterhaltungsprogramme (außer Fußballwettkämpfen) 
kaum genannt werden, werden Musikprogramme doch von einem Teil der Befragten verfolgt; 155 Seelsorger geben klassische Musik und/oder das leichtere Genre als regelmäßig gehört an. Daneben liegt das Schwergewicht vor allem auf Nachrichten und Berichten, Aktualitäten und religiösen Programmen. Letztere Programmsparte wird noch von 67, auch mit anderen Programmen kombiniert, genannt.

Tageszeitung: Die 312 befragten Priester lesen regelmäßig 741 Tageszeitungen, was 238 regelmäßig gelesene Tageszeitungen auf 100 Pfarrer bedeutet. Dies ist gut das Doppelte gegenüber der Angabe für die Niederlande im Durchschnitt: Nach dem Jahresbericht der niederländischen Tagespresse ${ }^{21}$ wurden im Jahre 1970 von je 100 Haushalten, einschließlich der Alleinstehenden, 107 Tageszeitungen gelesen. 80 Prozent der Priester lesen regelmäßig die katholischen Zeitungen „De Tijd" und/oder "De Volkskrant" bzw. ein überregionales Abend- und ein Morgenblatt aus einem katholischen Verlag. Neben einem oder mehreren überregionalen Blättern lesen 206 Befragte auch regelmäßig eine regionale Tageszeitung; 2 lesen weder ein überregionales Blatt noch eine regionale Tageszeitung regelmäßig; 27 lesen nicht regelmäßig eine überregionale Tageszeitung und $\mathbf{5 3}$ keine regionale Tageszeitung.

Trotz der Tatsache, daß der Geistliche als Seelsorger mehr Zeitungen liest als der Niederländer im Durchschnitt, verwendet er nicht mehr Zeit darauf als der durchschnittliche Zeitungsleser (45 Minuten). Das Lesen von drei oder mehr Zeitungen beansprucht also offenbar nicht mehr Zeit als das Lesen von zwei Zeitungen.

\section{Zeitaufwand und Zahl der gelesenen Tageszeitungen}

\begin{tabular}{l|c|c|c|c|c}
\hline & \multicolumn{3}{|c|}{$\begin{array}{c}\text { Zahl der Tageszeitungen, } \\
\text { die man regelmäßig liest }\end{array}$} & Gesamt; \\
Zeit, die man auf die & $\begin{array}{c}\text { eine } \\
\text { Zeitungen verwendet }\end{array}$ & $\begin{array}{c}\text { zwei } \\
\text { Tages- } \\
\text { zeitung }\end{array}$ & $\begin{array}{c}\text { Tages- } \\
\text { zeitungen }\end{array}$ & $\begin{array}{c}\text { dreh oder } \\
\text { zeitungen }\end{array}$ & Prozent- \\
werte & Gesamt; \\
absolut & \\
\hline 30 Min. od. weniger & 43,2 & 38,5 & 34,1 & 37,2 & 100 \\
31 Min. bis 45 Min. & 45,9 & 27,5 & 36,6 & 34,2 & 92 \\
mehr als 45 Min. & 10,8 & 33,9 & 29,3 & 28,6 & 77 \\
\hline Gesamt & 99,9 & 99,9 & 100 & 100 & $269^{*}$ \\
\hline
\end{tabular}

Diejenigen, die mehrere Zeitungen lesen, verwenden mehr Zeit auf das Zeitunglesen als jene, die nur eine Zeitung lesen, aber ein derartiger Unterschied ist nicht festzustellen $\mathrm{z}$ wischen jenen, die zwei Tageszeitungen lesen, und jenen, die drei oder mehr lesen.

* In die Tabelle sind nur die Beantworter aufgenommen, die eindeutig in die Kategorien "eine Tageszeitung“, „zwei Tageszeitungen“ und "drei oder mehr Tageszeitungen“ fielen. 
Es besteht ein deutlicher Zusammenhang zwischen der Zahl Zeitungen, die man regelmäßig liest, und der Wohnsituation: 75 Prozent der Pfarrer $(=90)$, die mit zwei oder mehr Priestern zusammenwohnen, lesen "De Tijd“ und "De Volkskrant" und gegebenenfalls noch eine weitere überregionale Tageszeitung; bei jenen, die mit einem Priester zusammenwohnen, beträgt dieser Prozentsatz 53 Prozent (35) und für die Alleinwohnenden 29 Prozent (21).

Der Umfang der Arbeitswoche beeinflußt nicht die auf das Zeitunglesen verwendete Zeit.

Der harte Kern des Leseverhaltens des Seelsorgers wird gebildet durch die Rubriken: Religion, redaktioneller Kommentar, lokale und regionale Nachrichten, Leserbriefe und Innen/Außenpolitik. Mehr unterhaltende Rubriken und Themen wie Comic Strips, Feuilleton, Bunte Seite wie auch Wirtschaft/Finanzen, Gerichtsberichte und Anzeigen werden wenig oder nicht als regelmäßig gelesen genannt.

„Meinungsblätter": Von den 6 als Meinungsblättern zu charakterisierenden Wochenblättern wird von den Seelsorgern vor allem "De Nieuwe Linie“, fortschrittlichkatholisch geprägt, regelmäßig gelesen, kombiniert (oder auch nicht) mit einem anderen Meinungsblatt (27 Prozent). 60 Prozent lesen unregelmäßig ein Meinungsblatt. Die Priester weichen hierbei nicht von einer (vergleichbaren) niederländischen Gruppe mit (mittlerer und) höherer Berufsbildung ab: nach TON 197022 lesen 40 Prozent dieser Gruppe regelmäßig ein Meinungsblatt (mindestens 75 Prozent der erschienenen Nummern); der Prozentsatz aller männlichen Personen von 13 Jahren ab und älter, die 75 Prozent der Nummern lesen, beträgt nach TON 1970 nur 9 Prozent. Altere Seelsorger lesen weniger Meinungsblätter als jüngere: 73 Prozent im Alter von 55 Jahren und mehr verfolgen ein Meinungsblatt nur unregelmäßig, in der mittleren Alterskategorie sind es 59 Prozent und in der jüngeren 50 Prozent.

Die Wohnsituation ist auf das Lesen von Meinungsblättern ohne Einfluß. Die Zeit, die man auf das Lesen von Meinungsblättern verwendet, ist sehr unterschiedlich: 21 Prozent verwenden eine halbe Stunde oder weniger darauf und 21 Prozent mehr als $11 / 2$ Stunden.

Film, Bücher und Zeitschriften: Von den befragten Priestern besuchten 78 (25 Prozent) ein- oder zweimal im abgelaufenen Jahr ein Kino und 104 (33 Prozent) dreimal oder öfter. Jüngere Pfarrer waren dabei weitaus aktiver als die älteren.

Auf die Frage, wie viele Bücher, die sich auf Religion, Kirche und Pfarramt beziehen oder Lebensanschauung betreffen, die Seelsorger im vergangenen Monat gelesen haben, gab es folgende Antworten:

(8 gaben keine Antwort)

24 Prozent hatten kein Buch gelesen,

41 Prozent ein oder zwei Bücher,

24 Prozent drei oder vier Bücher,

8 Prozent fünf oder mehr Bücher.

Außerdem hatten 42 Prozent der Seelsorger noch weitere Bücher gelesen. Jüngere lesen mehr Bücher über die genannten Themen als andere. Es besteht keine direkte Beziehung zwischen der Anzahl der gelesenen Bücher und der auf die Arbeit verwendeten Zeit. Wohl besteht eine Tendenz, das Lesen von Büchern über die vorgenannten Themen mehr oder weniger in die Schätzung des Umfangs ihrer Arbeitswoche einzubeziehen. 
Der Pfarrer scheint vor allem ein belesener Mann zu sein. Außer Büchern, Zeitungen und Meinungsblättern liest er noch regelmäßig eine nicht zu unterschätzende Anzahl von Zeitschriften. Anhand einer Liste von 29 Zeitschriften, von denen angenommen werden konnte, daß Geistliche sie lesen, wurde gefragt, ob diese regelmäßig gelesen würden. Die Liste umfaßte allgemein kirchliche Blätter sowie das Bistumsblatt, kirchliche und theologische Fachzeitschriften, und zwar wissenschaftliche wie Pastoralblätter, schließlich allgemeine Zeitschriften mit oder ohne religiöse Ausrichtung. Am häufigsten wurden das Bistumsblatt und die "Analecta" genannt, und zwar von 60 bzw. 85 Prozent der Befragten. Als Gruppe werden die vorgelegten 8 Fachzeitschriften am meisten als regelmäßig gelesen aufgeführt. Von 312 Beantwortern $811 \mathrm{mal}$.

Insgesamt wurde von den 312 Priestern $2156 \mathrm{mal}$ eine Zeitschrift als regelmäßig gelesen genannt. Durchschnittlich kommen auf jeden fast 7 Titel; darunter sind 2 allgemein kirchliche Blätter und 2 bis 3 Fachzeitschriften.

Obrigens führt dieser Zeitschriftenkonsum nicht dazu, daß der Seelsorger sich auch auf der Höhe des nötigen Informationsstandes fühlt über das, was in dem Bistum, in dem er tätig ist, vor sich geht: 24 Prozent meinen, daß sie durch die kirchliche Obrigkeit ihres Bistums über die von ihr gewünschte Führung ungenügend informiert werden, 57 Prozent finden die Information ausreichend, und 17 Prozent beurteilen sie fallweise als gut.

Die klassischen Medien kommen in der Leistungsbeurteilung durch die Priester schlecht weg: Die Hälfte und mehr finden die Berichterstattung durch Zeitungen, Hörfunk und Fernsehen über „die Kirche in den Niederlanden“, „den Priester" und „die zentrale Verwaltung der Kirche in Rom" nicht objektiv.

\section{Zusammenfassung}

Der Priester in der praktischen Seelsorge weicht in seinem Umgang mit den Massenmedien stark ab von den Menschen, für die er tätig ist, und zwar was den Umfang, die inhaltlichen Aspekte und auch die Gesamtheit seines Rezeptionsverhaltens angeht. Mit den eigenen Erfahrungen, die er in Konfrontation mit dem Angebot der Massenmedien erworben hat, steht der Seelsorger (verhältnismäßig) isoliert und marginal den Menschen gegenüber, für die er arbeitet: er kann seine direkten Erfahrungen nur mit seinen Kollegen und Hausgenossen austauschen, während die Menschen, für die er tätig ist, ihre Erfahrungen mit ihrem Pfarrer nicht wirklich teilen können. Der Priester (als "statistische Figur") gehört nicht zum "gewöhnlichen Publikum" (dem die Menschen, für die er arbeitet, beinahe alle angehören); die Unterhaltungsfunktion der Massenkommunikation geht an ihm fast vollständig vorbei; Fachliteratur, Arbeit, Versammlungen, Gespräche und Gesprächsgruppen treten an die Stelle des zuvor skizzierten Empfangsspielraums. Im Umgang mit den Massenmedien gehört der Seelsorger nicht zu „seinen“ Menschen, ja kaum zu seinen Artgenossen mit (mittlerer und) höherer Ausbildung. Der Priester läßt keine intellektuell bedingten elitären Vorurteile gegen Fernsehen, Hörfunk, Film und Bildberichte erkennen. In seinem gesamten Rezipientenverhalten ist jedoch eine deutliche Ausrichtung auf das gedruckte Wort festzustellen.

Der Umgang des Seelsorgers mit den Massenmedien erweckt stark den Eindruck, in direktem Zusammenhang mit seiner Auffassung von seiner eigenen Aufgabe, Funktion und Berufsausübung zu stehen, und mit den Problemen, die er selbst dabei hat: Wenn er das Fernsehen einschaltet, Radio hört, Zeitungen und Zeitschriften liest, 
dann nicht, um die Erfahrungen, Gefühle, Frustrationen, Erwartungen, kurzum die Reaktionen der Menschen auf das massenkommunikative Angebot teilen und begleiten zu können, sondern um gemäß der eigenen Auffassung fachkundig auftreten zu können, rundum informiert zu sein, und zwar aus eigenem beruflichen Interesse. Nebenbei sei hier vermerkt, daß weder die hierarchieorientierte Berichterstattung innerhalb des Bistums noch auch die Berichterstattung durch die klassischen Medien über Angelegenheiten, die den Seelsorger direkt angehen, ein uneingeschränkt günstiges Urteil bei ihm finden.

Der Priester steht, was seinen Umgang mit den Massenmedien angeht, in gewisser Weise im Abseits, er teilt in diesem Punkt nicht das Leben seiner Mitmenschen.

\section{Diskussion}

Bedeutet die Tatsache, daß die Lebenswelt des Seelsorgers hinsichtlich des Umgangs mit den Massenmedien tiefgehend verschieden ist von der Lebenswelt der Menschen, für die er arbeitet, nun auch, daß er den Menschen bei seiner Verarbeitung und Interpretation der durch die Massenmedien vermittelten Wirklichkeit nicht begleiten kann? Klar ist jedenfalls, daß seine mögliche Begleitung sich kaum auf eigene Erfahrung stützen kann. Nun ist die durch die Massenmedien vermittelte Wirklichkeit nicht völlig anders als auf andere Weise vermittelte Wirklichkeit: eigene Kenntnis, Empathie, soziale Intelligenz, Fachwissen und Erfahrung rüsten den Priester (möglicherweise) in ausreichendem Maße aus, um Gesprächspartner zu sein bei Angelegenheiten, die er nur vom Lesen, Hörensagen oder überhaupt nicht kennt. Obendrein ist es (möglicherweise) nicht die Aufgabe des Seelsorgers, die Erfahrungen anderer in den eigenen Erfahrungen wirklichkeitsgetreu widerzuspiegeln: Verständnishaltung bedeutet nicht per definitionem Gleichheit in allem, und nicht "Peyton Place ${ }^{\text {, }}$, sondern das Evangelium ist das Rationale für „religiös agogisches Handeln“.

Das Problem jedoch, das sich hier stellt, liegt nicht in bewußter, sondern in unbewußter Ungleichheit, nicht in der Isolierung als gewähltem Leben, sondern als gewachsenem Verhalten.

\section{Anmerkungen:}

1. Dieser Aufsatz basiert auf Daten aus einer PASTOMAC (Pastor en Massacommunicatie) genannten Untersuchung, die mehr Einsicht in die Beziehung zwischen den in der Basisseelsorge arbeitenden Priestern und verschiedenen Formen der Massenkommunikation verschaffen will. Die Untersuchung wurde vom Katholiek Instituut voor Massamedia (K.I.M.) in Zusammenarbeit mit dem Katholieke Radio Omroep (K.R.O.) initiiert und vom Instituut voor Massacommunicatie (I.M.C.) der Katholischen Universität Nimwegen in den Niederlanden durchgeführt, während das Meinungsforschungsinstitut Intomart die Feldarbeit und die primäre Datenverarbeitung besorgte. Ein $\mathrm{Z}_{\text {wischenbericht über einige Daten }}$ ist erschienen unter dem Titel: Debeuckelaere, G.P., \& Evers, W.J.M.: Pastomac: Pastor en Massacommunicatie; I.M.C., Nijmegen, März 1971. Ein ausführlicher Bericht ist betitelt: Debeuckelaere, G.P., \& Evers, W.J.M.: Pastor en Risiko, I.M.C., Nijmegen, März 1972.

2. Dieser Terminus wurde erstmalig verwendet von: De Fleur, M.L., \& Larssen, O.N.: The Flow of Information, New York 1958; vgl. auch: De Fleur, M.L.: Theories of Mass Communication, New York 1966.

3. Der Terminus stammt von: Fearing, F.: Social Impact of the Mass Media of Communication, in Henry, N.B. (Hrsg.): Mass Media and Education, Chicago 1954, S. 165-191. 
Vgl. auch: Fearing, F.: Toward a Psychological Theory of Human Communication, in: Journal of Personality “, 1953, XXII, S. 71-88. Fearing, F.: Human Communication, in: "Audio Visual Communication Review“, 1962, X, 5, S. 78-108.

4. Vgl. hierzu u. a.: Davison, W.P.: On the Effects of Communication, in: „Public Opinion Quarterly“, 1959, S. 343-360; Bauer, R.A.: The Obstinate Audience, in: "American Psychologist", 1964, S. 319-328; Bauer, R.A.: Communication as Transaction, in: Payne, D.E.: The Obstinate Audience, Ann Arbor 1965, S. 3-11; Klapper, J.T.: The Effects of Mass Communication, Glencoe Ill. 1966; Klapper, J.T.: Mass Communication, An Old Way Resurveyed, in: „Public Opinion Quarterly“, 1963, S. 515-527; vgl. auch: Dröge, F., Weissenhorn, R., \& Haft, H.: Wirkungen der Massenkommunikation, Münster 1969; vgl. für andere Untersuchungskompilationen über Massenkommunikation: Tannebaum, P., Greenberg, B.: Mass Communication, in: „Annual Review of Psychology“, 1968, 19, S. 351-386; Weiß, W.: Effects of Mass Media Communication, in: Lindzey, G. \& Aronson, E. (Hrsg.): Handbook of Social Psychology, Boston 1969.

5. Katz, E., \& Lazarsfeld, P.F.: Personal Influence, The Part Played by the People in the Flow of Mass Communications, Glencoe Ill. 1955.

6. Vgl. hierzu: Brouwer, M.: Stereotypen als folklore, Vinkeveen 1968.

7. Der Durchschnittsniederländer widmet sich 2 bis 2,5 Stunden täglich dem Fernsehen: "Luisteren en Kijken '70/71", Abteilung Studium und Forschung der Nederlandse Omroep Stichting (N.O.S.), S. 4; der Durchschnittsniederländer im Alter von 12 Jahren und mehr hörte im Jahre 1971 täglich (zwischen 7.00 Uhr vormittags und $19.00 \mathrm{Uhr}$ nachmittags) 3 Stunden Hörfunk. Hörerdichte 4. Quartal 1971 (2. Teil), Bericht Nr. 71, Abteilung Studium und Forschung des N.O.S. März 1972, S. 8; die niederländischen Tageszeitungen werben für sich selbst mit dem aus der Untersuchung hervorgegangenen Resultat, daß der Durchschnittsniederländer pro Tag durchschnittlich 45 Minuten Zeitung liest. - Die entsprechenden Zahlen für die B.R. Deutschland (1970): Fernsehen 1:53 h, Hörfunk 1:11 h, Zeitung $0: 30 \mathrm{~h}$. Nach einer Infratest-Untersuchung referiert in „ZV+ ZV“ 1972, S. 914 (Die Nutzung der Freizeit).

8. Stephenson, W.: The Play Theory of Mass Communication, Chicago 1967.

9. Stephenson, W.: a.a.O., S. 2.

10. Vgl. für diese und weitere Daten aus PASTOMAC: Debeuckelaere, G.P., \& Evers, W.J.M.: a.a.O.

11. Die Untersuchungsgruppe ist hinsichtlich ihrer Ausbildung als homogen anzusehen, während das Niveau mehr oder minder der Ausbildung eines Kandidaten der Theologie gleichgesetzt werden kann. Die Untersuchungsgruppe wird deshalb insgesamt als der Kategorie der Menschen mit der höchsten Ausbildung zugehörig angesehen.

12. "Luisteren en Kijken '70/71": a.a.O., S. 4.

13. Dies ist aus der relativen Sehdichte aller Niederländer im Alter von 12 Jahren und mehr mit mittlerer oder höherer Ausbildung abzuleiten: „Intern Bulletin“ 72-33 CPO/TV a. Nr. 18 v. 26. April 1972, Continu-Programma-Onderzoek T.V., 4. Quartal 1971, Abteilung Studium und Forschung des N.O.S., April 1972, S. 9.

14. Der Zuschauerkreis des NTS Journaal, Abteilung Studium und Forschung NRU/NTS 1965; Continu-Programma-Onderzoek WTV - 33, 28-4967, Abtcilung Studium und Forschung NRU/NTS, 1967; Continu-Programma-Onderzock Televisic, Zusammenfassung von Ergebnissen 1967, 4. Quartal, Bericht Nr. 25, Abteilung Studium und Forschung NRW/NTS.

15. "Späte ${ }^{\alpha}$ Zuschauer sind jene, die gewöhnlich erst spät am Abend nach 21.00 Uhr fernsehen. Pfarrer, die nur Zeitabschnitte vor 21.00 Uhr nannten, wurden als "frühe" $\mathrm{Zu}$ schauer angesehen. Pfarrer, die nur Zeiten nach 21.00 Uhr nannten, wurden als "späte“ Zuschauer betrachtet. Der Befragte konnte auf die diesbezügliche Frage mehr als eine Antwort geben.

16. „Luisteren en Kijken '70/71“: a.a.O., S. 6.

17. A.a.O.

18. A.a.O., S. 8.

19. Diese Angaben stammen aus einem nicht veröffentlichten Bericht. Sie werden bestätigt durch Angaben über die Sehzeitaufteilung der verschiedenen Bildungsniveaus auf die Programm-Kategorien: Information, Kultur, erzählende Unterhaltung, Unterhaltung, Sport und Jugendprogramme: die Kategorie der Menschen mit mittlerer und höherer Ausbildung folgen in ihrer Sehzeitaufteilung auf die verschiedenen Programm-Kategorien getreu der Sehzeitverteilung der gesamten niederländischen Bevölkerung. „Intern Bulletin“, $72-33 \mathrm{CPO} / \mathrm{TV}$ a. Nr. 18: a.a.O., S. 14. 
20. Hörerdichte, 4. Quartal 1971 (2. Teil) Bericht Nr. 71 : a.a.O., S. 8.

21. De Nederlandse Dagbladpers, Jahresbericht 1970, Amsterdam 1971, S. 17.

22. TON $=$ Tijdschriftenlezerskring Onderzoek Nederland 1970, Nederlandse Onderneming voor Uitgevers Marktonderzoek, N.O.V.U.M. N.V. 1970.

\section{S U M M A R Y}

Three Catholic Dutch Communications Institutes, (,Katholiek Instituut voor Massamedia;, ,Katholieke Radio Omroep' and ,Instituut voor Massacommunicatie of the Catholic University of Nijmegen) together untertook a socio-empirical study of the attitude of Catholic priests in the Netherlands towards Mass communications. The survey reveals that parish priests, vicars and chaplains use media in a basically different way from the ordinary Dutch citizen, and so have a different approach from that of their own parishoners. Priests have much less time to spend on TV and Radio. Dailies, periodicals and especially professional magazines are read more frequently and more intensively than by the average citizen. The results of the survey lead to the question as to whether priests are somewhat isolated through their exposure to mass media. Are they still able to keep in touch with their people through and about their media experiences? On only one point do their interests seem to coincide when they are viewing football matches.

\section{RES U MÉ}

Le ,Katholiek Instituut voor Massamedia', le ,Katholieke Radio Omroep' et le ,Instituut voor Massacommunicatie de la Faculté Catholique de Nimègue (Pays-Bas) ont réalisé ensemble une enquête socio-empirique sur le comportement des prêtres (aux Pays-Bas) face à la communication de masse. Cette enquête eut pour résultat que les prêtres, les vicaires et les chapelains utilisent les moyens de communication de masse en bien des points différemment de la moyenne de la population des Pays-Bas, cela signifie donc: également differemment de la moyenne des membres de leurs communautés paroissiales. Les prêtres passent beaucoup moins de temps à regarder la télévision et à écouter la radio. Cependant, ils lisent la plupart du temps et de façon plus intensive que la moyenne de la population les quotidiens, les périodiques et particulièrement les revues spécialisées. Les résultats de cette enquête amènent à se poser la question suivante: les prêtres, se trouvent-ils dans un certain isolement du fait de leur comportement différent par rapport aux moyens de communication de masse? Peuvent-ils s'entretenir convenablement des évènements avec les membres de leurs communautés. Leur comportement est identique en un seul point: au cours des retransmissions télévisées des matchs de football.

\section{RES UMEN}

El „Katholiek Instituut voor Massamedia“, la „Katholieke Radio Omroep“ y el „Instituut voor Massacommunicatie ${ }^{\alpha}$ de la universidad católica de Nimega (Holanda) han llevado a cabo en común un análisis social-empírico sobre el comportamiento de sacerdotes católicos con cura de almas (en Holanda) respecto de los medios de comunicación social. El análisis llevó a la conclusión de que párrocos, vicarios y coadjutores utilizan los medios de comunicación social en puntos esenciales de modo muy diverso a como lo hace, por termino medio, el resto de la población holandesa, e incluso de modo distinto a como lo hacen por lo general los miembros de sus feligresías. Los sacerdotes dedican bastante menos tiempo a la televisión y la radio. Por el contrario prestan mayor atención y leen con mayor intensidad que el promedio normal de la población diarios, revistas y sobre todo revistas especializadas. Los resultados del citado análisis plantean necesariamente ciertos interrogantes: ¿̇ Se encuentran los sacerdotes con cura de almas, a causa de su peculiar relación con los medios de comunicación social (exposure to the mass media) en un cierto aislamiento? ¿Están en condiciones de hablar con suficiente conocimiento de causa sobre programas de televisión ó radio con los fieles de su parroquia? Solo en una materia es idéntico su comportamiento: respecto de las retransmisiones televisadas de partidos de futbol. 